\title{
SEMANTIC CONTEXT-BASED ON-DEMAND SERVICE MODEL FOR LAND COVER CHANGE DETECTION
}

\author{
Huaqiao Xing ${ }^{1}$, Dongyang Hou ${ }^{2}$ \\ ${ }^{1}$ School of Surveying and Geo-informatics, Shandong Jianzhu University, Jinan, China - xinghuaqiao@126.com, \\ ${ }^{2}$ School of Geosciences and Info Physics, Central South University, Changsha, China - houdongyang1986@163.com
}

Commission IV, ICWG IV/III, WG IV/4

KEY WORDS: Change Detection, Semantic Context, On-demand Service, Service Composition

\begin{abstract}
:
Land cover change (LCC) detection is widely used in many social-benefit areas, such as land cover updating, sustainable development and geographical situation monitoring. With the development of Web Services and cloud computing, a number of remote sensed algorithms and models have been published as web services. An on-demand service is urgent to be generated by compositing a sequence of atomic services, according to different situations. Context information plays an important role in automatic service composition. Traditional context information models mainly focus on service only, and ignore the relationships among users and services. To address this problem, we introduce the service context and user context into the context information. OWL-SC and OWL-UC are then proposed by extending the traditional service description model (i.e., OWL-S). Finally, a contextaware on-demand service model for LCC detection is built to realize service composition and optimization.
\end{abstract}

\section{INTRODUCTION}

Land cover change (LCC) detection is the process of identifying land change areas and types by using remote sensed images acquired in different time (HUSSAIN et al., 2013; Tewkesbury et al., 2015). In recent years, with the emergence and development of Web Services and cloud computing, it becomes an important trend to introduce the theory and method of service computing into the field of LCC detection, for realizing intelligent extraction, networked collaboration, knowledge-based services and on-demand processing of land cover change data (Xing et al., 2019). LCC detection is a complex geo-processing task involving with different steps, such as pre-processing, change information extraction and change type determination. During last thirty decades, a large number of LCC detection algorithms or models have been proposed to address land cover change requirements. However, these algorithms or models often have their own advantages and limitations, these exist no single one can be universal for any cases. Therefore, it is an argent practical problem to build ondemand LCC detection service according to different situations under a dynamical environment.

In order to address such complicated geoprocessing requirement, automatic web service composition is an effective way to create service chain by composing a collection of atomic services (Cruz et al., 2012). It is can be seen as the process of searching the appropriate services based on users' requirement (i.e., input and output data). The critical point is the service match by utilizing syntactic and semantic description of web services. In the geospatial community, the IOPE (input, output, precondition and effect) information of OWL-S is most frequently-used method to judge whether the services can be correctly connected. For example, Yue et al. (2007) proposed an approach to the automatic composition of geospatial Web services based on the geospatial semantics with OWL-S (Peng et al., 2007). However, the existing IOPE of services is only reflect the semantic information of themselves, with less consideration the context information of relationships between services and end-user's requirements. Therefore, the traditional methods may generate service chain that differ from a user's intention. In fact, context information plays an important role in the process of service composition. Its influence is mainly reflected in two aspects(Cuddy et al., 2005). One is the interaction between users and web services, the appropriate services or composite services can be selected according to the context information of users. The other is the business logic within the service composition, which can be described as service relations.

LCC detection has unique domain characteristics of data diversity, operation complexity and user demand diversification (Chen et al., 2013). First, the data types involved in LCC detection are diverse from different sensors with different spatial resolutions, spectral resolutions, temporal resolutions and spectral resolutions. Second, coupling degree between services and services is high, business logic is closely related, and a certain type of LCC detection service often uses different algorithms or processes in its implementation. Although the functions are similar, the algorithms are different, that is, they have their specific scope of application. Third, with different professional backgrounds, end-users often have different application requirement. By analysing the characteristics of these areas, it is not difficult to see that the LCC detection service domain contains a variety of context information, such as user context information including user needs and preferences, service context information including the relationship between services and service applicability.

In recent years, several efforts have been made in the contextbased service composition. For example, Niu et al. (2011) presented a multi-granularity context model to strengthen the flexibility and intelligence of dynamic service composition(Niu et al., 2011). Cao et al. (2015) propose a context-aware automatic service composition method, which reduces the number of failures and composition time(Cao et al., 2015). However, the current context-based research of geographic information service discovery and matching technology has not effectively considered the user context (e.g., users' intention and preferences), and the service context information (e.g., 
relationship between services and service applicability), and cannot fully describe the context information. In addition, the current research on the context of service-oriented composition lacks an effective formal description method, which may hander the process of dynamic service composition.

To address the above challenges, we propose a novel approach of semantic context-based on-demand LCC detection service model. A context information model is firstly constructed from the aspects of user context and service context. Then, by extending the traditional service semantic language OWL-S, a formal expression method is presented with OWL-UC and OWL-SC, which is used to describe user context and service context, respectively. Additionally, a context-aware on-demand service model is finally proposed to composite services to an on-demand service chain based on semantic context description and matching.

\section{SEMANTIC DESCRIPTION OF CONTEXT-BASED WEB SERVICE}

Context model is a conceptual model describing context elements and their relationships in specific application scenarios. The impact of context on Web services is mainly reflected in two aspects. One is the user information, which mainly manifested in the information interaction with the user, according to the user's current context information to choose the appropriate data to display. For example, an end-user expects the final change result is change area, he or she can choose change area detection service as the core processing service. The other is the internal business logic, which is referred to relationships among the control flow in a specific workflow. Therefore, context information is built from service context and user context, respectively.

\subsection{OWL-SC based service context}

Service context for service composition refers to the relationship between a service and other services in service composition and its scope of application, which is can be represented as Service Relation (SR) and Service Application (SA). Service context has an important impact on the internal business logic of service composition. Different service context may generate different service composition schemes. Traditional service context is context information based on quality of service (QoS) description, which is separated from service description model and is not conducive to service consistency description. In this paper, service context is integrated into service description model, and service context information is no longer a single one. At present, the Capabilities of Web Processing Service (WPS) remain at the grammatical level of service input and output parameters, and cannot provide semantic information about services. Although Ontology Web Language for Services (OWL-S) can provide a unified and standard language structure for Web services and provide rich semantic expression capabilities, the current OWL$\mathrm{S}$ description mainly focuses on service input and output parameters, service categories, premises and results, and cannot fully describe the context information of services. Especially in the field of LCC detection, there is a lack of service correlation and service applicability description. Therefore, there are some limitations in using these service description languages for dynamic service composition. To solve this problem, this paper extends the service ontology description language OWL-S, adds service relationship and service applicability description to the description model, and proposes a formal description method based on OWL-SC (Web Ontology Language for Service Context), as shown in Figure 2. Based on the three original subclasses of OWL-S, Service Profile, Service Model and Service Grounding, the framework adds service context classes including service relationship and service applicability description.

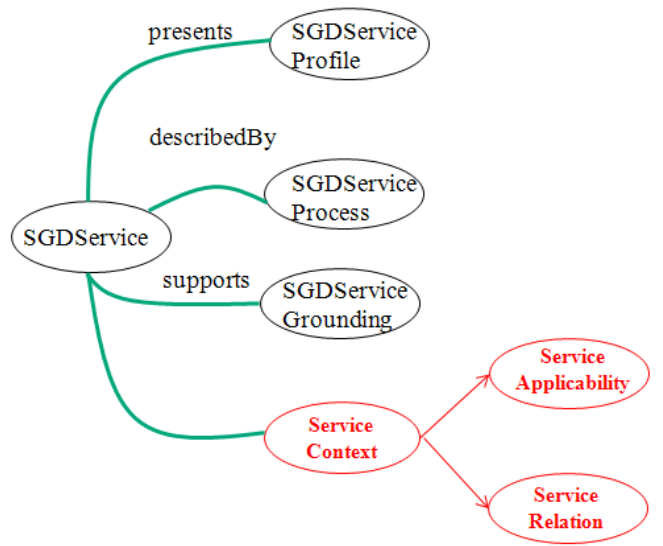

Fig.1. OWL-SC based service context description

LCC detection service involves a variety of data types. Although the functions of a function of LCC detection service are similar, there are differences in implementation methods. It often does not have the ability to process all types of remote sensed data, that is to say, it has its specific scope of application. This paper mainly describes service applicability from two aspects: the applicability of input data, the applicability of relationship between input data and output data. It can be expressed by a two tuple: $\mathrm{SA}=\{\mathrm{IA}, \mathrm{OA}\}$. IA and $\mathrm{OA}$ represent the applicability of service to data type and attributes of input data and output data, respectively.

\subsection{OWL-UC based user context}

User Context expresses users' requirements and preferences for services, including functional and non-functional. It mainly includes four parts: user needs, user preferences, context and user-defined rules, which can be represented by a tuple: $\mathrm{UC}=$ $\{$ Req, Pre, UR $\}$. Req refers to the functional requirements of users, including the requirements for input and output data. Pre refers to users' preferences for services, including the format of output data and the time consumed by services. UR refers to user-defined rules, for example, forest experts who only want to know the change of forest, may select services with higher detection of forest change through user-defined rules. Aiming at the user context in the field of surface coverage updating, a formal description method based on OWL (Web Ontology Language User Context) ontology description language is proposed as shown in Figure 2.

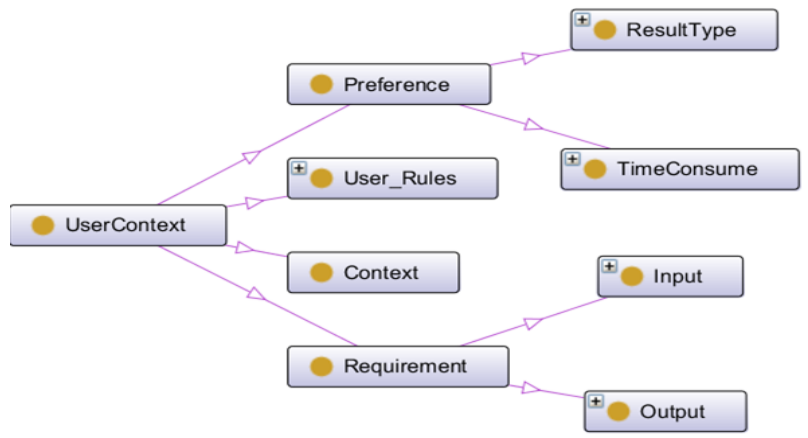

Fig.2. OWL-UC based user context description 


\section{CONTEXT-AWARE ON-DEMAND SERVICE MODEL}

On the basis of context modelling in the field of LCC detection service, an on-demand service model is proposed, as shown in Figure 3. The model mainly includes the following modules:

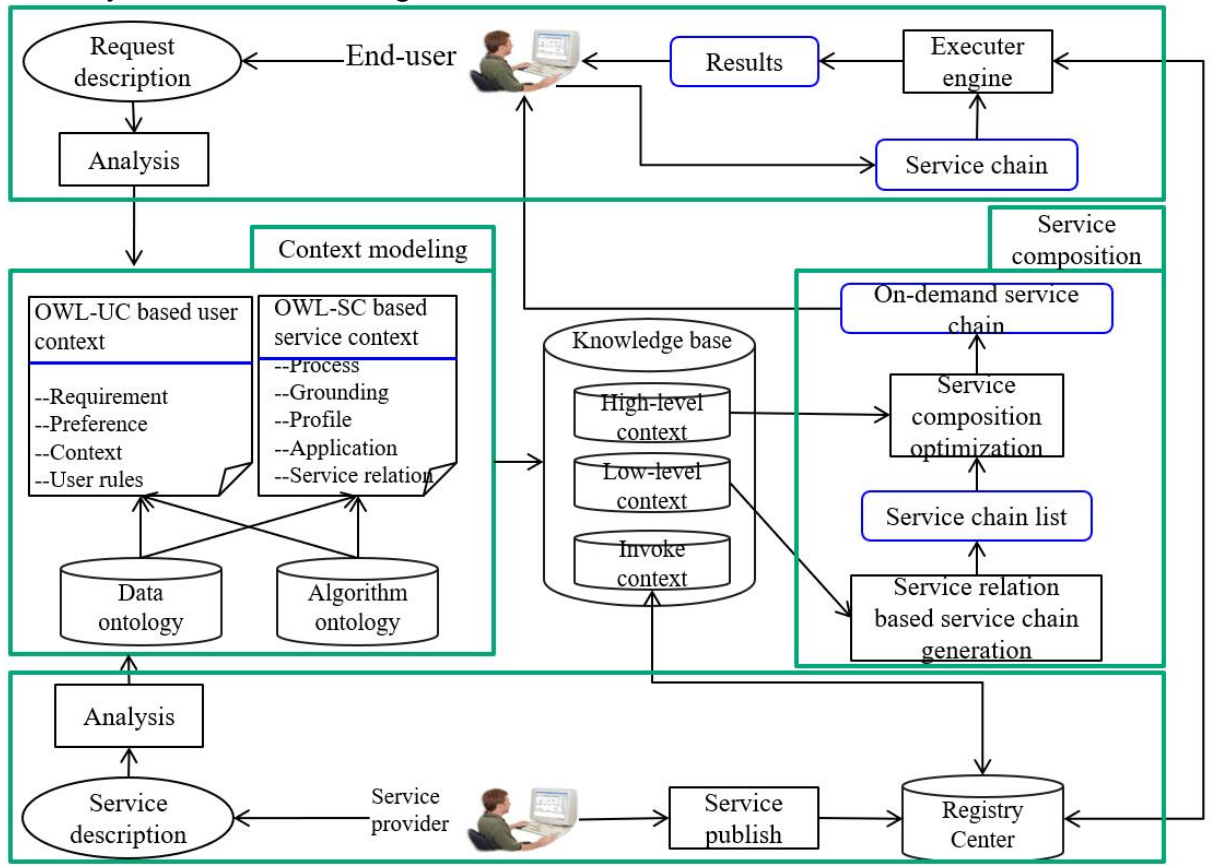

Fig.3 On-demand service model based on semantic context

(1) User request

Users request description mainly includes two parts. One is functional description with input data and expected output data description. The other is non-functional description with user context, user rules and preferences.

\section{(2) Service register}

Service providers register services through the service registration interface. On the one hand, service addresses and service profile descriptions are provided to service registries according to standards. On the other hand, service relationships and service applicability descriptions are parsed into service context.

\section{(3) Context modelling}

Based on OWL-UC, the user's request information is modelled and formally expressed. Based on OWL-SC, the service context information is modelled and formally expressed.

\section{(4) Service chain generation}

Firstly, service composition path is dynamically generated based on service relation according to user requirements and service relation context, and service composition scheme set is generated. In this scheme set, there may be several combination schemes satisfying user requirements. Then, the optimal value of service composition is calculated according to user preferences and service applicability context, and the combination scheme set is arranged and returned to users on demand.

\section{(5) Service composition optimization}

Users can rearrange the set of service composition schemes returned by the system according to their experience. They can also adjust the services in the service composition. They can customize the service composition schemes that are most suitable for their own conditions, and then send the final scheme to the execution engine. The execution engine invokes the corresponding services through the invocation context of OWL-SC to perform the final operation, and the execution node will be executed. The results are finally returned to the user.

\section{CONCLUSION}

LCC detection services contain a variety of contextual information, which play an important role in on-demand service composition. This paper analyses the domain characteristics of LCC detection service, builds service context and user context model for service composition respectively. Furthermore, we extend the formal description languages OWL-S and OWL to formalize them. An ervice context model based on OWL-SC and a user context model based on OWL-UC are constructed. On this basis, an on-demand service composition model for LCC detection service is proposed. By modelling and formalizing the context, it can provide a basis for dynamic service composition and service composition optimization.

\section{REFERENCE}

Cao, Z., Zhang, X., Zhang, W., Xiong, X., Shi, J., Xu, H., 2015. A Context-Aware Adaptive Web Service Composition Framework, IEEE International Conference on Computational Intelligence \& Communication Technology.

Chen, J., Hao, W.U., Songnian, L.I., Liao, A., Chaoying, H.E., Peng, S., 2013. Temporal logic and operation relations based 
knowledge representation for land cover change web services. Isprs Journal of Photogrammetry \& Remote Sensing 83, 140150 .

Cruz, S.A.B., Monteiro, A.M.V., Santos, R., 2012. Automated geospatial Web Services composition based on geodata quality requirements. Computers \& Geosciences 47, 60-74.

Cuddy, S., Katchabaw, M., Lutfiyya, H., 2005. Context-aware service selection based on dynamic and static service attributes, IEEE International Conference on Wireless \& Mobile Computing.

HUSSAIN, Masroor, Chen, D., CHENG, Angela, Wei, H., STANLEY, David, 2013. Change detection from remotely sensed images: From pixel-based to object-based approaches. Isprs Journal of Photogrammetry \& Remote Sensing 80, 91106.

Niu, W., Gang, L., Zhao, Z., Hui, T., Shi, Z., 2011. Multigranularity context model for dynamic Web service composition tr. Journal of Network \& Computer Applications 34, 312-326.

Peng, Y., Di, L., Yang, W., Yu, G., Zhao, P., 2007. Semanticsbased automatic composition of geospatial Web service chains. Computers \& Geosciences 33, 649-665.

Tewkesbury, A.P., Comber, A.J., Tate, N.J., Lamb, A., Fisher, P.F., 2015. A critical synthesis of remotely sensed optical image change detection techniques. Remote Sensing of Environment 160, 1-14.

Xing, H., Chen, J., Wu, H., Hou, D., 2019. A Web ServiceOriented Geoprocessing System for Supporting Intelligent Land Cover Change Detection. ISPRS International Journal of GeoInformation 8, 50. 